\title{
Abkürzungs- und Schrifttumsverzeichnis
}

a. A.

$\mathrm{aaO}$

ABl.

abl.

Abs.

AcP

ADAC

ADSp.

a. E.

ÄndG

a. F.

AFG

AG

AHB

AKB

Alt.

Amtl. Begr.

Amtl. Bek.

Amtsbl.

Anh.

Anl.

AnwBl.

AO

AOK

AP

AR

ArbG

ArchivPF

Art.

AtomG

AUB

Aufl.

AuR

AuslPflvG

AVG

AVK

BAG

BAGE

BAT

Baumbach/

Lauterbach anderer Ansicht

am angegebenen Ort

Amtsblatt

ablehnend

Absatz

Archiv für die civilistische Praxis

Allgemeiner Deutscher Automobilclub

Allgemeine Deutsche Spediteurbedingungen

am Ende

Änderungsgesetz

alte Fassung

Arbeitsförderungsgesetz v. 25. 6. 1969 (BGBI. I 582)

Amtsgericht

Allgemeine Haftpflichtversicherungsbedingungen

Allgemeine Bedingungen für die Kraftfahrversicherung

Alternative

Amtliche Begründung

Amtliche Bekanntmachung

Amtsblatt

Anhang

Anlage

Anwaltsblatt

Abgabenordnung

Allgemeine Ortskrankenkasse

Nachschlagwerk des Bundesarbeitsgerichts (Arbeitsrechtliche Praxis)

Automobil-Rundschau

Arbeitsgericht

Archiv für das Post- und Fernmeldewesen

Artikel

Gesetz über die friedliche Verwendung der Kernenergie und den Schutz gegen ihre Gefahren i. d. F. v. 31. 10. 1976 (BGBl. I 3053)

Allgemeine Unfallversicherungsbedingungen

Auflage

Arbeit und Recht, Zeitschrift für Arbeitsrechtspraxis

Gesetz über die Haftpflichtversicherung für ausländische Kraftfahrzeuge und Kraftfahrzeuganhänger v. 24. 7. 1956 (BGBI. I 667, ber. BGBI. 1957 I 368)

Angestelltenversicherungsgesetz v. 20.12.1911 (RGBl. 989)

Allgemeine Versicherungsbedingungen der privaten Krankenversicherung

Bundesarbeitsgericht

Sammlung der Entscheidungen des Bundesarbeitsgerichts

Bundesangestelltentarifvertrag

Baumbach/Lauterbach ${ }^{46}$ Zivilprozeßordnung (1988) mit Name des Bearbeiters 
Abkürzungs- und Schrifttumsverzeichnis

BayBgm.
BayGemZ
BayObLG
BayObLGSt

BayObLGZ

\section{BayStrWG \\ BayVGH \\ BayZ \\ BB \\ BBahn \\ BBahnG \\ BBG}

Bd.

BeamtVG

Becker/Böhme

Beil.

betr.

Betrieb

BFH

BFHE

BFStrG

BG

BGB

BGBl.

BGH

BGHSt.

BGHWarn.

\section{BGHZ}

BKGG

Blutalkohol

Böhmer

Böhmer (SHG)

BOKraft

BOStrab

BRDrucks.

BRRG

Bruck/Möller

BSG

BSGE

BSHG

BStB1.

BTDrucks.

Buchst.

Büro

BVerfG

BVerfGE

BVerwG
Zeitschrift „Der Bayerische Bürgermeister“

Bayerische Gemeindezeitung

Bayerisches Oberstes Landesgericht

Entscheidungen des Bayerischen Obersten Landesgerichts in Strafsachen (Neue Folge seit 1951, die älteren Jahrgänge werden mit der Bandzahl zitiert)

Entscheidungen des Bayerischen Obersten Landesgerichts in Zivilsachen (Neue Folge seit 1951, die älteren Jahrgänge werden mit der Bandzahl zitiert)

Bayerisches Straßen- und Wegegesetz

Bayerischer Verwaltungsgerichtshof

Zeitschrift für Rechtspflege in Bayern (1905-1934)

Zeitschrift „Der Betriebsberater“

Zeitschrift "Die Bundesbahn“

Bundesbahngesetz

Bundesbeamtengesetz

Band

Beamtenversorgungsgesetz v. 24. 8. 1976 (BGBl. I 2485, 3839)

Becker/Böhme Kraftverkehrshaftpflichtschäden ${ }^{17}$ (1989)

Beilage

betreffend

Zeitschrift „Der Betrieb“

Bundesfinanzhof

Sammlung der Entscheidungen und Gutachten des Bundesfinanzhofs

Bundesfernstraßengesetz

Zeitschrift "Berufsgenossenschaft"

Bürgerliches Gesetzbuch

Bundesgesetzblatt

Bundesgerichtshof

Sammlung der Entscheidungen des Bundesgerichtshofs in Strafsachen

Rechtsprechung des Bundesgerichtshofs in Zivilsachen, soweit nicht in BGHZ enthalten, Fortsetzung von WarnR (seit 1961)

Sammlung der Entscheidungen des Bundesgerichtshofs in Zivilsachen

Bundeskindergeldgesetz i. d. F. v. 21. 1. 1982 (BGBl. I 14)

Zeitschrift „Blutalkohol“

Böhmer Das Reichshaftpflichtgesetz (1950)

Böhmer Das Sachschadenhaftpflichtgesetz (1954)

Verordnung über den Betrieb von Kraftfahrunternehmen im Personenverkehr v. 21. 6. 1975 (BGBl. I 1573)

Betriebsordnung für Straßenbahnen v. 11. 12. 1987 (BGBl. I 2648)

Drucksache des Deutschen Bundesrats

Beamtenrechtsrahmengesetz

Bruck/Möller Versicherungsvertragsgesetz ${ }^{8}$ (1983) mit Namen des Bearbeiters

Bundessozialgericht

Entscheidungen des Bundessozialgerichts

Bundessozialhilfegesetz

Bundessteuerblatt, Teil I, II oder III

Drucksachen des Deutschen Bundestags, geordnet nach Wahlperioden (seit 1949)

Buchstabe

Zeitschrift „Das juristische Büro“

Bundesverfassungsgericht

Entscheidungen des Bundesverfassungsgerichts

Bundesverwaltungsgericht 
Abkürzungs- und Schrifttumsverzeichnis

BVerwGE

BVG

DAR

DAVorm

DB

Deutsch

d. h.

DIN

DJ

DJT

DJZ

DNotZ

DÖV

DR

Drees/Kuckuk/

Werny

DRiZ

DRiZRspr.

Drucks.

DRZ

DVB1

EBO

Eckelmann

EE

EGBGB

EGZPO

EheG

1. EheRG

\section{EKMR}

Erl.

Erman

ErwG

Esser/Schmidt

EurGRZ

EuGVÜ

EvBl.

EVO

EWG

f

FamRZ

ff

Filthaut

Fn.

Full

FV
Entscheidungen des Bundesverwaltungsgerichts

Bundesversorgungsgesetz i. d. F. v. 22. 1. 1982 (BGBl. I 22)

Deutsches Autorecht, Rechtszeitschrift des ADAC, München

Der Amtsvormund

Deutsche Bundesbahn

Deutsch Haftungsrecht Erster Band: Allgemeine Lehren (1976)

das heißt

Deutsche Industrienorm

Deutsche Justiz, Zeitschrift

Deutscher Juristentag

Deutsche Juristenzeitung

Deutsche Notar-Zeitschrift

Zeitschrift „Die öffentliche Verwaltung“

Zeitschrift „Deutsches Recht“ (1931 - 1942)

Drees/Kuckuk/Werny Straßenverkehrsrecht ${ }^{5}$ (1985)

Deutsche Richterzeitung

Beilage „Rechtsprechung“ zu DRiZ (1925- 1935 und 1951 - 1965)

Drucksache

Deutsche Rechts-Zeitschrift (1946-1950)

Zeitschrift „Deutsches Verwaltungsblatt“

Eisenbahn-Bau- und Betriebsordnung

Eckelmann Schadensersatz für Kraftfahrzeugschäden ${ }^{3}$ (1974)

Eisenbahn- und verkehrsrechtliche Entscheidungen und Abhandlungen (1885-1935)

Einführungsgesetz zum BGB

Einführungsgesetz zur Zivilprozeßordnung

Ehegesetz v. 20. 2. 1946 (AblKR 77)

Erstes Gesetz zur Reform des Ehe- und Familienrechts v. 14.6. 1976 (BGBl. I 549)

Europäische Kommission für Menschenrechte

Erläuterung

Erman Handkommentar zum Bürgerlichen Gesetzbuch ${ }^{7}$ (1981) mit Name des Bearbeiters

Gesetz über die erweiterte Zulassung von Schadenersatzansprüchen bei Dienst- und Arbeitsunfällen v. 7. 12. 1943 (RGBl. I 674)

Esser/Schmidt Schuldrecht Allgemeiner Teil ${ }^{6}$ (1984)

Europäische Grundrechte-Zeitschrift

Übereinkommen der Europäischen Gemeinschaft über die gerichtliche Zuständigkeit und die Vollstreckung gerichtlicher Entscheidungen in $\mathrm{Zi}$ vil- und Handelssachen (BGBI. 1972 II 774)

Evidenzblatt der Rechtsmittelentscheidungen (Wien, seit 1934)

Eisenbahnverkehrsordnung

Europäische Wirtschaftsgemeinschaft

und folgende Seite

Zeitschrift „Ehe und Familie im privaten und öffentlichen Recht" (seit 1954)

und folgende Seiten

Haftpflichtgesetz ${ }^{2}$ (1988)

Fußnote

Full Zivilrechtliche Haftung im Straßenverkehr (1980)

Finanzvertrag (BGBI. 1955 II 381) 
Abkürzungs- und Schrifttumsverzeichnis

GAL

Geigel

GG

ggf.

GKG

Gottwald

Greger

GrS

Gruch.

GüKG

GVBl.

GVG

HaftpfiG

Hainmüller

Halbs.

HansGZ

HansRGZ

HansRZ

HessVGH

HESt.

HEZ

HGB

HGrG

Himmelreich/

Klimke

h. L.

h. M.

Hofmann

HRR

Gesetz über eine Altershilfe für Landwirte v. 14. 9. 1965 (BGBl. I 1449)

Geigel Der Haftpflichtprozeß ${ }^{19}$ (1986)

Grundgesetz für die Bundesrepublik Deutschland

gegebenenfalls

Gerichtskostengesetz

Gottwald Schadenszurechnung und Schadensschätzung (1979)

Greger Beweis und Wahrscheinlichkeit (1978)

Großer Senat

Gruchot, Beiträge zur Erläuterung des deutschen Rechts (1857 - 1933)

Güterkraftverkehrsgesetz

Gesetz- und Verordnungsblatt (Landesrecht)

Gerichtsverfassungsgesetz

Haftpflichtgesetz

Hainmüller Der Anscheinsbeweis und die Fahrlässigkeitstat im heutigen deutschen Schadensersatzprozeß (1966)

Halbsatz

Hanseatische Gerichtszeitung (1880-1927)

Hanseatische Rechts- und Gerichtszeitschrift (1928 - 1943)

Hanseatische Rechtszeitschrift (1918 - 1927)

Verwaltungsgerichtshof des Landes Hessen

Höchstrichterliche Entscheidungen in Strafsachen (1948 - 1949)

Höchstrichterliche Entscheidungen in Zivilsachen (1948 - 1950)

Handelsgesetzbuch

Haushaltsgrundsätzegesetz v. 19. 8. 1969 (BGBl. 1969 I 1273, 1277)

Kfz-Schadensregulierung ${ }^{4}$ (Fortführungsstand 1989)

herrschende Lehre

herrschende Meinung

Hofmann Haftpflichtrecht für die Praxis (1989)

Höchstrichterliche Rechtsprechung, Vereinigte Entscheidungssammlung der bisherigen Rspr. der Oberlandesgerichte, HöchstRR und JR Rspr., Verlag Walter de Gruyter \& Co, Berlin (1928 - 1942)
i. d. F.
i. e. S.
i. d. $R$.
IntAbk
IntVO
IPRax
IPRspr.

in der Fassung

im engeren Sinn

in der Regel

Internationales Abkommen für den Verkehr mit Kraftfahrzeugen

Verordnung über den internationalen Kraftfahrzeugverkehr

Praxis des internationalen Privat- und Verfahrensrechts

Die deutsche Rechtsprechung auf dem Gebiete des internationalen Privatrechts

i. S. d.

im Sinne des (der)

i. V. m.

in Verbindung mit

Jagusch/Hentschel Jagusch/Hentschel Straßenverkehrsrecht ${ }^{30}$ (1989)

JherJb.

JP

Jherings Jahrbücher der Dogmatik des bürgerlichen Rechts (1857-1942)

JR

Zeitschrift "Juristische Praxis“

JRPrV

Juristische Rundschau

JurZentr.

JuS

Justiz

Juristische Rundschau für die Privatversicherung (1924 - 1943)

Mitteilungen der juristischen Zentrale des ADAC

Zeitschrift „Juristische Schulung“

Zeitschrift "Die Justiz“, Amtsblatt des Justizministeriums Baden-Württemberg

JW

Juristische Wochenschrift $(1872-1939)$

JZ

Juristenzeitung 
Abkürzungs- und Schrifttumsverzeichnis

KF

KFG

KG

Krumme

KVO

KVR

LAG

Lange

Larenz

LFZG

LG

Lkw

LM

LS

LSE

LSG

LuftVG

LuK

LZ

Maassen

MdE

MDR

MedR

Mertens

MünchKomm

m. w. Nachw.

NATO
NdsRpfl.
n. F.
NJ
NJW
NJW-RR
NTS
NTS-AG
NVwZ
NZA
NZV
ÖJZ
ÖRZ
Österr. OGH
OGH
OGHSt.
OGHZ
OHG

Karlsruher Forum

Kraftfahrzeuggesetz

Kammergericht

Krumme Straßenverkehrsgesetz (1977)

Kraftverkehrsordnung

Kraftverkehrsrecht von A bis Z, herausgegeben von Dr. Weigelt, Loseblattausgabe

Landesarbeitsgericht

Lange Schadensersatz (1979)

Larenz Lehrbuch des Schuldrechts Allgemeiner Teil ${ }^{14}$ (1987) und Besonderer Teil ${ }^{12}(1981)$

Lohnfortzahlungsgesetz v. 27. 7.1969 (BGB1. I 946)

Landgericht

Lastkraftwagen

Nachschlagewerk des Bundesgerichtshofs, Loseblattsammlung herausgegeben von Lindenmaier, Möhring u. a.

Leitsatz

Lexikon straßenverkehrsrechtlicher Entscheidungen, herausgegeben v.

Günther Xanke

Landessozialgericht

Luftverkehrsgesetz

Zeitschrift "Luft- und Kraftfahrt"

Leipziger Zeitschrift für Deutsches Recht (1907-1933)

Maassen Beweisprobleme im Schadensersatzprozeß (1975)

Minderung der Erwerbsfähigkeit

Monatsschrift für Deutsches Recht

Zeitschrift „Medizinrecht“

Mertens Der Begriff des Vermögensschadens im bürgerlichen Recht (1967)

Münchner Kommentar zum Bürgerlichen Gesetzbuch ${ }^{2}$ (1984ff) mit Name des Autors

mit weiteren Nachweisen

North Atlantic Treaty Organization

Zeitschrift "Niedersächsische Rechtspflege"

neue Fassung oder neue Folge

Zeitschrift „Neue Justiz“

Neue Juristische Wochenschrift

Neue Juristische Wochenschrift - Rechtsprechungsreport

NATO-Truppenstatut v. 19.6. 1951 (BGBl. 1961 II 1190)

Ausführungsgesetz zum NATO-Truppenstatut v. 18.8. 1961 (BGBI. II 1183)

Neue Zeitschrift für Verwaltungsrecht

Neue Zeitschrift für Arbeitsrecht

Neue Zeitschrift für Verkehrsrecht

Österreichische Juristenzeitung

Österreichische Richterzeitung (1904-1938 und seit 1954)

Oberster Gerichtshof für Österreich

Oberster Gerichtshof für die britische Zone

Entscheidungen des OGH in Strafsachen $(1949-1950)$

Entscheidungen des OGH in Zivilsachen (1949-1950)

Offene Handelsgesellschaft 
Abkürzungs- und Schrifttumsverzeichnis

OLG

OLGSt.

OLGZ

OVG

OWiG

Palandt

PBefG

PflVG

Pkw

PostG

PostReiseO

Pr. allg. LR

Prölss/Martin

PrOVG

Prütting

RdA

RdK

RdL

Recht

RG

RGBI.

RGRKomm.

RGRspr.

RGWarn.

RGSt

RGZ

RHaftpflG

RKnappschG

ROHG

Rosenberg/Schwab

Rpfleger

Rspr.

Rüth/Berr/Berz

RuS

RVO

S.

s.

s. a.

Sanden/Völtz

SchlHA

Schloën/Steinfeltz

Schneider

SchwbG

SchwJZ

schwSVG
Oberlandesgericht

Entscheidungen der Oberlandesgerichte zum Straf- und Strafverfahrensrecht

Entscheidungen der Oberlandesgerichte in Zivilsachen

Oberverwaltungsgericht

Ordnungswidrigkeitengesetz

Palandt Bürgerliches Gesetzbuch ${ }^{48}$ (1989), mit Name des Bearbeiters

Personenbeförderungsgesetz v. 21. 3. 1961 (BGBl. I S. 241)

Pflichtversicherungsgesetz

Personenkraftwagen

Gesetz über das Postwesen v. 28. 7. 1969 (BGBl. I S. 1006)

Postreiseordnung

Preußisches allgemeines Landrecht

Prölss/Martin Versicherungsvertragsgesetz ${ }^{24}$ (1988)

Preußisches Oberverwaltungsgericht (1877-1941), zitiert nach Band und Seite

Prütting Gegenwartsprobleme der Beweislast (1983)

Zeitschrift „Recht der Arbeit“

Das Recht des Kraftfahrers, Zeitschrift (1926- 1943 und 1949-1955)

Zeitschrift „Recht der Landwirtschaft“

Zeitschrift "Das Recht"

Reichsgericht

Reichsgesetzblatt

Reichsgerichtsrätekommentar zum BGB ${ }^{12}$ (1976ff), mit Name des Bearbeiters

Rechtsprechung des RG in Strafsachen (1879-1888)

Rechtssprechung des Reichsgerichts, herausgegeben von Warneyer (1908 - 1943)

Sammlung der Entscheidungen des Reichsgerichts in Strafsachen (nach Band und Seite)

Sammlung der Entscheidungen des Reichsgerichts in Zivilsachen (nach Band und Seite)

Reichshaftpflichtgesetz

Reichsknappschaftsgesetz v. 23. 6.1923 (RGBl. I 431)

Reichsoberhandelsgericht (Entscheidungen 1871-1880)

Rosenberg/Schwab Zivilprozeßrecht ${ }^{14}$ (1986)

Zeitschrift „Der deutsche Rechtspfleger" (1931 - 1944 und seit 1948)

Rechtsprechung

Rüth/Berr/Berz Straßenverkehrsrecht ${ }^{2}$ (1988)

Zeitschrift „Recht und Schaden“

Reichsversicherungsordnung

Seite

siehe

siehe auch

Sanden/Völtz Sachschadenrecht des Kraftverkehrs ${ }^{5}$ (1986)

Schleswig-Holsteinische Anzeigen, Justizministerialblatt für SchleswigHolstein (n. F. seit 1837)

Schloën/Steinfeltz Regulierung von Personenschäden (1978)

E. Schneider Beweis und Beweiswürdigung ${ }^{4}$ (1987)

Schwerbehindertengesetz

Schweizerische Juristenzeitung (1904ff)

Schweizerisches Straßenverkehrsgesetz v. 19. 12. 1958 (SR 741.01) 
Abkürzungs- und Schrifttumsverzeichnis

SeuffA

SG

SGB

$\mathrm{SGb}$

SHG

SJZ

Soergel

SoldG

Staudinger

Stein/Jonas

StGB

Stiefel/Hofmann

StPO

StrG

StVG

StVo

StVZO

SVG

SZ

Thomas $/$ Putzo

TÜV

u. a.

u. U.

v.

VAE

Venzmer

VersR

VersWiss.

VerwArch.

VerwRspr.

VG

VGH

VGT

VkBl.

VkRdsch.

VM

VN

VO

Vorb.

VP
J. A. Seufferts Archiv für Entscheidungen der obersten Gerichte in den deutschen Staaten $(1847$ - 1944)

Sozialgericht

Sozialgesetzbuch (mit Angabe des Buches in römischer Ziffer)

Zeitschrift „Die Sozialgerichtsbarkeit“"

Gesetz über die Haftpflicht der Eisenbahnen und Straßenbahnen für Sachschäden

Süddeutsche Juristenzeitung (1946 - 1950)

Soergel Bürgerliches Gesetzbuch mit Einführungsgesetz und Nebengesetzen ${ }^{11}$ (1978ff), mit Name des Autors

Soldatengesetz v. 19. 8. 1975 (BGBl. I 2273)

Staudinger Kommentar zum Bürgerlichen Gesetzbuch ${ }^{12}$ (1983), mit Name des Bearbeiters

Zivilprozeßordnung ${ }^{20}$ (1977ff)

Strafgesetzbuch

Stiefel/Hofmann Kraftfahrtversicherung ${ }^{13}$ (1986)

Strafprozeßordnung

Straßengesetz (Ländergesetze)

Straßenverkehrsgesetz

Straßenverkehrsordnung

Straßenverkehrs-Zulassungs-Ordnung

Soldatenversorgungsgesetz v. 26.7.1957 in der Fassung der Bek. v. 21. 4. 1983 (BGBl. I 457)

Entscheidungen des österreichischen Obersten Gerichtshofs in Zivil- und Justizverwaltungssachen

Thomas/Putzo Zivilprozeßordnung ${ }^{15}$ (1987)

Technischer Überwachungsverein

und andere, unter anderem

unter Umständen

vom

Verkehrsrechtliche Abhandlungen und Entscheidungen (1936-1944)

Venzmer Mitverursachung und Mitverschulden im Schadensersatzrecht (1960)

Zeitschrift "Versicherungsrecht"

Zeitschrift "Versicherungswissenschaft, Versicherungspraxis und Versicherungsmedizin“ (1947-1950, seitdem „Deutsche Versicherungszeitschrift für Sozialversicherung und Privatversicherung")

Zeitschrift „Verwaltungsarchiv“ (1893-1942 und seit 1957)

Verwaltungsrechtsprechung in Deutschland, Sammlung oberstrichterlicher Entscheidungen aus dem Verfassungs- und Verwaltungsrecht

Verwaltungsgericht

Verwaltungsgerichtshof

Deutscher Verkehrsgerichtstag (Veröffentlichung der gehaltenen Referate und erarbeiteten Empfehlungen durch die Deutsche Akademie für Verkehrswissenschaft)

Verkehrsblatt

Zeitschrift "Verkehrsrundschau“"

Zeitschrift "Verkehrsrechtliche Mitteilungen“"

Der Versicherungsnehmer, Zeitschrift

Verordnung

Vorbemerkung

Zeitschrift „Die Versicherungspraxis“ (1903-1943 und seit 1950) 
Abkürzungs- und Schrifttumsverzeichnis

VR

VRS

VVG

VW

VwGO

VwV-StVO

Wahrendorf
Walter
WarnJ
WarnRspr.
WHG
WJ
WM
Wussow
Wussow/Küppers-
busch

ZA

ZAkDR

ZBISozVers

ZBR

ZDG

ZfS

ZfV

ZMR

Zöller

ZPO

ZRP

zust.

zutr.

ZVkWiss.

ZVersWiss.

ZVR

zW.

ZZP
Verkehrsrechtliche Rundschau (1921 - 1944)

Verkehrsrechtssammlung (zitiert nach Band und Seite)

Gesetz über den Versicherungsvertrag

Versicherungswirtschaft, Halbmonatsschrift der deutschen Individualversicherung

Verwaltungsgerichtsordnung

Allgemeine Verwaltungsvorschrift zur Straßenverkehrsordnung v. 24. 11. 1970 (VkBl. 1970 758, berichtigt VkB1. 197130 , zuletzt geändert VkBl. 1980 520)

Wahrendorf Die Prinzipien der Beweislast im Haftungsrecht (1976)

Walter Die Haftung des Kraftfahrzeughalters (1962)

Walter Die Regulierung des Kraftfahrzeugschadens ${ }^{4}$ (1973)

Jahrbuch der Entscheidungen zum BGB und den Nebengesetzen, begründet v. Warneyer $(1900-1938)$

Rechtsprechung des RG (siehe RGWarn.)

Wasserhaushaltsgesetz

Informationen zum Versicherungs- und Haftpflichtrecht, herausgegeben von Wussow

Zeitschrift „Wertpapiermitteilungen"

Wussow Das Unfallhaftpflichtrecht ${ }^{13}$ (1985)

Wussow Systematik des Haftpflichtrechts (1958)

Wussow/Küppersbusch Ersatzansprüche bei Personenschaden ${ }^{4}$ (1986)

Zusatzabkommen v. 3. 8. 1959 zum NATO-Truppenstatut (BGBI. 1961 II 1218)

Zeitschrift der Akademie für Deutsches Recht

Zentralblatt für Sozialversicherung

Zeitschrift für Beamtenrecht (1929-1943 und seit 1953)

Zivildienstgesetz i. d. F. v. 29.9. 1983 (BGBl. I 1221, 1370)

Zeitschrift für Schadensrecht

Zeitschrift für Versicherungswesen

Zeitschrift für Miet- und Raumrecht

Zöller Zivilprozeßordnung ${ }^{15}$ (1987), mit Name des Bearbeiters

Zivilprozeßordnung

Zeitschrift für Rechtspolitik

zustimmend

zutreffend

Zeitschrift für Verkehrswissenschaft (1923 - 1944 und seit 1948)

Zeitschrift für die gesamte Versicherungswissenschaft (1901-1943 und seit 1960 )

Zeitschrift für Verkehrsrecht (Österreich)

zweifelhaft

Zeitschrift für Zivilprozeß (1879- 1943 und seit 1950) 